Original Article

\title{
Visceral fat rating is a useful indicator in risk assessment among coronary artery disease patients treated with aggressive lipid lowering therapy
}

\author{
Aniket Puri*, Vatsal Singh, Saurabh Pandey, Charu Kant Singh, \\ Sunny R.K. Singh
}

King George’s Medical University, Lucknow, Uttar Pradesh 226003, India

\section{A R T I C L E I N F O}

\section{Article history:}

Received 28 May 2013

Accepted 20 November 2013

Available online 3 January 2014

Keywords:

Impedance

Coronary

Dyslipidemia
Visceral fat

\begin{abstract}
A B S T R A C T
Background: Visceral fat rating (VFR), calculated by bioelectrical impedance, is a new parameter associated with obesity and Coronary Artery Diseases (CAD). We have attempted to assess population of treated CAD patients classified based on VFR.

Methods: We enrolled consecutive patients having documented CAD who had received prior treatment along with high-dose lipid lowering drugs for a minimum of 6 wks and compared with healthy controls. CAD patients with VFR $>12$ were labeled as High Visceral fat CAD (HVC) while those with VFR $\leq 12$ were grouped as Low Visceral fat CAD (LVC). Established anthropometric indices like waist circumference (WC) and Body Mass Index (BMI) were measured. We used bioelectrical impedance to measure VFR using a device called InnerScanV (TANITA, Tokyo). A complete lipid profile was recorded. Groups were compared by chi-square, t-test and one-way analysis of variance. Multivariate analysis was done for identifying risk factors.

Results: 303 subjects were enrolled, including $150 \mathrm{CAD}$ patients and 153 healthy controls. Among CAD group, there were 74 (49.3\%) HVC and 76 (51.7\%) LVC patients. On comparing treated HVC and treated LVC patients, HVC patients had expectedly higher WC, BMI and higher Triglycerides (TG), total cholesterol (TC) and LDL cholesterol. On comparing the patients with healthy controls, HVC group again had higher WC, BMI, LDL, TC:HDL ratio, LDL:HDL ratio and lower HDL levels. On the other hand, LVC patients had a lower BMI than the healthy controls and there was no difference in the lipid parameters, apart from lower HDL in LVC patients, between the two groups. VFR along with WC, HDL, LDL:HDL and TC:HDL were independent risk factors among treated CAD patients. WC, HDL, LDL:HDL and TC:HDL ratios were risk factors among LVC patients while in addition to these, BMI and LDL were extra risk factors among HVC patients and thus associated with VFR.

Conclusion: High visceral fat is associated with deranged anthropometric measurements and lipid profile. We hypothesize that HVC patients might be resistant to conventional treatment, while LVC patients respond well to aggressive lipid lowering therapy. HVC patients are also associated with more risk factors than LVC patients.

Copyright @ 2013, INDIACLEN. Publishing Services by Reed Elsevier India Pvt Ltd. All rights
\end{abstract} reserved.

\footnotetext{
* Corresponding author.

E-mail address: aniketpuri@hotmil.com (A. Puri).

2213-3984/\$ - see front matter Copyright @ 2013, INDIACLEN. Publishing Services by Reed Elsevier India Pvt Ltd. All rights reserved. http://dx.doi.org/10.1016/j.cegh.2013.11.002
} 


\section{Introduction}

At present, the population at risk of Coronary Artery Diseases (CAD) is recognized by various criteria, for example the presence of metabolic syndrome, which includes the conventional risk factors like hypertension, diabetes, hyperlipidemia and increases waist circumference or obesity. Abdominal obesity is also proposed to be the major contributor of associated health risks. Notably, visceral fat, rather than the subcutaneous part of abdominal fat, is considered the marker of 'dysfunctional adipose tissue' and has emerged of central importance. ${ }^{1}$ It has also become evident that the accumulation of visceral fat not only accompanies but antedates the onset of the components of the metabolic syndrome and related disorders, e.g., insulin resistance, hypertension and coronary heart disease. Recently, with the advent of Bioelectrical Impedance Analysis (BIA) technique, direct and accurate estimate of visceral fat has become possible. ${ }^{2}$ However, the association of visceral fat with $\mathrm{CAD}$ has not been studied extensively yet. Therefore, in our present study we have attempted to analyze the treated patients of Coronary Artery Disease (CAD) on the basis of their visceral fat rating (VFR) measured by BIA. We aim to study VFR as a risk factor along with conventional CAD risk factors in treated CAD patients. We further intend to study the profile of treated CAD patients population subdivided based on VFR and find association of conventional risk factors in each subdivision.

\section{Methods}

This is a cross-sectional observation study. We enrolled consecutive patients attending as out-patients at the Cardiology Department. Subjects were categorized into 2 groups; documented CAD patients and normal healthy individuals as controls. Documented CAD patients included had a history of Acute ST elevation or Non-ST elevation Myocardial Infarction; or Chronic stable angina with treadmill test positive or coronary angiography proven $\mathrm{CAD}$ and having received prior treatment with high-dose lipid lowering therapy (typically atorvastatin $\geq 40 \mathrm{mg} / \mathrm{d}$ ) for at least the prior 6 weeks. The documented CAD patients were further subdivided into High Visceral fat CAD (HVC) and Low Visceral fat CAD (LVC) patients. HVC group includes CAD patients with VFR $>12$ and LVC includes CAD patients with VFR $\leq 12$. VFR greater than 12 is considered abnormal and signifies the presence of high visceral fat. The healthy individuals included those without documented CAD or being at high risk for the same, i.e., those not qualifying as Metabolic Syndrome based on the NCEPATPIII criteria ${ }^{3}$ or having Framingham 10 years risk score ${ }^{4}$ of less than $10 \%$ and not on any lipid lowering therapy. These independent healthy subjects were recruited from OPD staff, unrelated accompaniments of CAD patients and patients who visited the OPD for routine check-ups or other ailments.

Exclusion criteria included all subjects younger than 35 years or older than 75 years, those having cardiac pacemaker or other metallic implants in body, patients of neuromuscular or skeletal disorders and limb anomalies. After an informed written consent, a detailed history about smoking, alcohol intake, previously diagnosed diabetes or hypertension, drug intake and family history for CAD was taken. Anthropometric indices of weight, height and waist circumference (WC) were measured and Body Mass Index (BMI) was calculated. A fasting blood sample for complete lipid profile comprising of triglycerides (TG), total cholesterol (TC), low density lipoprotein (LDL) and high density lipoprotein (HDL) was done at the Department of Biochemistry. For lipids levels, we referred to NCEP-ATPIII Guidelines. ${ }^{5}$ According to these standard guidelines, hypercholesterolemia is defined as TC $>200 \mathrm{mg} / \mathrm{dl}$, LDL$\mathrm{C}>100 \mathrm{mg} / \mathrm{dl}, \mathrm{TG}>150 \mathrm{mg} / \mathrm{dl}$ and HDL-C $<40 \mathrm{mg} / \mathrm{dl}$ in males or $\mathrm{HDL}-\mathrm{C}<50 \mathrm{mg} / \mathrm{dl}$ in females. Dyslipidemia is defined as one or more abnormal parameter.

We used InnerScanV (TANITA Inc, Tokyo), which works on the principal of BIA, to measure VFR. The BIA device applies an alternating current to electrodes placed on subjects' hand and feet and yields a measure of body resistance and reactance. Body composition is estimated based on the specific resistivity offered by different body tissues.

\section{Statistical analysis}

Data were summarized as Mean \pm SE. In Tables 1 and 2, categorical parameters were analyzed using chi-square test while continuous parameters were analyzed by t-test. In Table 3, continuous data from three groups were analyzed by one-way analysis of variance (ANOVA). In Tables 4 and 5, multivariate analysis was performed with adjustment for all significant socio-demographic parameters i.e., age, sex, smoking, diabetes and hypertension. GraphPad Prism 3.0 software was used for chi-square, STATISTICA (Windows

\begin{tabular}{|c|c|c|c|}
\hline Characteristics & $\begin{array}{l}\text { Healthy } \\
\text { controls } \\
(n=153)\end{array}$ & $\begin{array}{l}\text { All CAD } \\
\text { patients } \\
(n=150)\end{array}$ & $p$-value \\
\hline Age (years) & $39.99 \pm 0.79$ & $57.88 \pm 0.92$ & $<0.0001$ \\
\hline Sex (males) & $64(41.8 \%)$ & 122 (81.3\%) & $<0.0001$ \\
\hline Smoking & 19 (12.4\%) & $61(40.7 \%)$ & $<0.0001$ \\
\hline Diabetes & $14(9.2 \%)$ & $78(52.05)$ & $<0.0001$ \\
\hline F. history & 13 (8.5\%) & $18(12.0 \%)$ & 1.012 \\
\hline Hypertension & 32 (20.9\%) & 68 (45.3\%) & $<0.0001$ \\
\hline Alcohol & $15(9.8 \%)$ & $24(16 \%)$ & 0.107 \\
\hline VFR & $7.77+0.26$ & $11.30 \pm 0.38$ & $<0.0001$ \\
\hline WC $(\mathrm{cm})$ & $85.88 \pm 0.78$ & $94.73 \pm 0.83$ & $<0.0001$ \\
\hline BMI $\left(\mathrm{kg} / \mathrm{m}^{2}\right)$ & $24.28 \pm 0.33$ & $23.96 \pm 0.35$ & 0.512 \\
\hline $\mathrm{TG}(\mathrm{mg} / \mathrm{dl})$ & $136.76 \pm 5.37$ & $140.63 \pm 4.65$ & 0.586 \\
\hline TC (mg/dl) & $158.21 \pm 3.42$ & $160.83 \pm 3.22$ & 0.577 \\
\hline HDL (mg/dl) & $39.47 \pm 0.97$ & $35.65 \pm 0.64$ & 0.001 \\
\hline LDL (mg/dl) & $91.38 \pm 3.08$ & $97.06 \pm 2.83$ & 0.211 \\
\hline TC/HDL & $4.32 \pm 0.13$ & $4.70 \pm 0.12$ & 0.064 \\
\hline LDL/HDL & $2.58 \pm 0.12$ & $2.86 \pm 0.10$ & 0.076 \\
\hline
\end{tabular}

$\mathrm{CAD}=$ Coronary Artery Disease; LVC = Low visceral fat $(\leq 12) \mathrm{CAD}$ patients; HVC $=$ High visceral fat $(>12)$ CAD patients; $W C=$ Waist Circumference, $\mathrm{BMI}=$ Body Mass Index; $\mathrm{TG}=$ Triglyceride, $\mathrm{TC}=$ Total Cholesterol; HDL = High Density Lipoprotein $; \mathrm{LDL}=$ Low Density Lipoprotein; BF\% = Body Fat Percentage; VFR = Visceral Fat Rating. 
Table 2 - Comparison of socio-demographic characteristics of HVC and LVC patients with healthy controls.

\begin{tabular}{|c|c|c|c|c|}
\hline \multirow[t]{2}{*}{ Parameters } & \multirow{2}{*}{$\begin{array}{c}\text { Healthy controls with } \\
\text { normal VFR }(n=145)\end{array}$} & \multicolumn{2}{|c|}{ CAD patients $(n=150)$} & \multirow[t]{2}{*}{$p$-value } \\
\hline & & LVC patients $(n=76)$ & HVC patients $(n=74)$ & \\
\hline Age (years) & $39.79 \pm 0.81$ & $56.43+1.33$ & $59.36+1.24$ & $<0.0001$ \\
\hline Sex (males) & $62(42.8 \%)$ & $56(73.7 \%)$ & $66(89.2 \%)$ & $<0.0001$ \\
\hline Smoking & 19 (13.1\%) & $32(42.1 \%)$ & $29(39.2 \%)$ & $<0.0001$ \\
\hline Diabetes & $13(8.9 \%)$ & $36(47.4 \%)$ & $42(56.8 \%)$ & $<0.0001$ \\
\hline F. history & $11(7.6 \%)$ & $7(9.2 \%)$ & $11(14.9 \%)$ & 0.314 \\
\hline Hypertension & 31 (21.4\%) & $28(36.8 \%)$ & 40 (54.1\%) & $<0.0001$ \\
\hline Alcohol & $15(10.3 \%)$ & $11(14.5 \%)$ & $13(17.6 \%)$ & 0.259 \\
\hline
\end{tabular}

version 8.0) was used for t-test and ANOVA and MINITAB 16 was used for binary logistic multivariate analysis. $p<0.05$ was considered statistically significant.

\section{Results}

We enrolled 303 subjects in 2 groups: 150 (49.5\%) patients of documented CAD and 153 (50.5\%) healthy controls. Documented CAD patients were further subdivided into 74 (49.3\%) High Visceral fat CAD (HVC) and 76 (51.7\%) Low Visceral fat CAD (LVC) patients. In Tables 2-5, we included only 145 subjects in healthy control group for analysis as 8 subjects from healthy control group who had VFR $>12$ were excluded from further analysis.

\subsection{Comparison between all treated CAD patients and healthy controls}

All socio-demographic characteristics of the groups are summarized in Table 1 . VFR of treated CAD patients was significantly higher than the healthy controls (11.3 vs $7.8 ; p<0.05$ ). The median VFR in the treated CAD group was 12 , which is also taken as the upper limit for normal VFR level. The median VFR for health controls was 8 . There was no difference among the anthropometric indices except WC, which was significantly higher in CAD patients. Lipid profile for all treated CAD patients and healthy controls were statistically similar except HDL which was significantly lower in the CAD patients (35.7 mg/dl vs $39.5 \mathrm{mg} / \mathrm{dl} ; p<0.05$ ).

\subsection{Comparison between HVC and LVC patient}

Socio-demographic characteristics of healthy controls have been compared with LVC and HVC patients in Table 2. Among the CAD group (Table 3) the WC and BMI of HVC patients was significantly higher than LVC patients $(100.6 \mathrm{~cm}$ vs $89 \mathrm{~cm}$ and $26 \mathrm{~kg} / \mathrm{m}^{2}$ vs $21.9 \mathrm{~kg} / \mathrm{m}^{2}$ respectively).

HVC patients had higher TG, TC and LDL values in comparison to LVC patients (152.7 vs $128.9 \mathrm{mg} / \mathrm{dl}, 171.5$ vs $150.5 \mathrm{mg} / \mathrm{dl}$ and $105.1 \mathrm{vs} 89.2 \mathrm{mg} / \mathrm{dl}$ respectively). However, there was no difference in HDL and the ratios of TC:HDL and LDL:HDL between the two groups (35.8 vs $35.5 \mathrm{mg} / \mathrm{dl}$, 5 vs 4.8 and 3 vs 2.7 respectively).

\subsection{Comparison of HVC and LVC subgroups with healthy controls}

When analyzing healthy controls along with LVC and HVC subgroups of CAD patients (Tables 2-5), we included only 145 subjects in the healthy control group for analysis as 8 subjects from healthy control group who had VFR $>12$ were excluded.

On comparing both CAD patients subgroups with the healthy controls (Table 3), expectedly both WC and BMI of HVC patients

Table 3 - Comparison of anthropometric characteristics and lipid profile of HVC and LVC patients with healthy controls.

\begin{tabular}{|c|c|c|c|}
\hline \multirow[t]{2}{*}{ Parameters } & \multirow{2}{*}{$\begin{array}{l}\text { Healthy controls with } \\
\text { normal VFR }(n=145)\end{array}$} & \multicolumn{2}{|c|}{ CAD patients $(n=150)$} \\
\hline & & LVC patients $(n=76)$ & HVC patients $(n=74)$ \\
\hline WC (cm) & $84.88 \pm 0.72$ & $89.01 \pm 1.09^{\mathrm{a}}$ & $100.61 \pm 0.82^{\mathrm{ab}}$ \\
\hline BMI $\left(\mathrm{kg} / \mathrm{m}^{2}\right)$ & $23.89 \pm 0.35$ & $21.93 \pm 0.40^{\mathrm{a}}$ & $26.04 \pm 0.45^{\mathrm{ab}}$ \\
\hline $\mathrm{TG}(\mathrm{mg} / \mathrm{dl})$ & $133.78 \pm 5.22$ & $128.88 \pm 6.39$ & $152.70 \pm 6.52^{\mathrm{b}}$ \\
\hline TC (mg/dl) & $157.29 \pm 3.53$ & $150.48 \pm 4.23$ & $171.47 \pm 4.59^{b}$ \\
\hline HDL (mg/dl) & $39.61 \pm 0.99$ & $35.51 \pm 1.03^{\mathrm{a}}$ & $35.78 \pm 0.76^{\mathrm{a}}$ \\
\hline LDL (mg/dl) & $91.89 \pm 3.08$ & $89.19 \pm 3.84$ & $105.14 \pm 3.97^{\mathrm{ab}}$ \\
\hline TC/HDL & $4.28 \pm 0.14$ & $4.47 \pm 0.17$ & $4.95 \pm 0.17^{\mathrm{a}}$ \\
\hline LDL/HDL & $2.57 \pm 0.12$ & $2.67 \pm 0.14$ & $3.04 \pm 0.14^{\mathrm{a}}$ \\
\hline
\end{tabular}


Table 4 - Multivariate analysis comparing healthy controls versus all CAD patients adjusted for all confounding socio-demographic characteristics.

\begin{tabular}{lcr} 
Parameters & $\begin{array}{c}\text { Odds ratio } \\
\text { (confidence interval) }\end{array}$ & $p$-value \\
\hline VFR & $1.21(1.08-1.36)$ & 0.001 \\
WC $(\mathrm{cm})$ & $1.12(1.06-1.18)$ & $<0.001$ \\
BMI $\left(\mathrm{kg} / \mathrm{m}^{2}\right)$ & $1.07(0.96-1.20)$ & 0.229 \\
TG $(\mathrm{mg} / \mathrm{dl})$ & $1.00(0.99-1.01)$ & 0.934 \\
TC $(\mathrm{mg} / \mathrm{dl})$ & $1.00(0.99-1.01)$ & 0.601 \\
HDL $(\mathrm{mg} / \mathrm{dl})$ & $0.92(0.88-0.97)$ & 0.001 \\
LDL $(\mathrm{mg} / \mathrm{dl})$ & $1.01(1.00-1.02)$ & 0.125 \\
TC/HDL & $1.49(1.16-1.92)$ & 0.002 \\
LDL/HDL & $1.61(1.19-2.17)$ & 0.002 \\
\hline LVC = Low visceral fat $(\leq 12)$ CAD patients; VFR = Visceral Fat \\
Rating; WC = Waist Circumference, BMI = Body Mass Index; \\
TG = Triglyceride, TC = Total Cholesterol; HDL = High Density Li- \\
poprotein; LDL = Low Density Lipoprotein. \\
\hline
\end{tabular}

were significantly higher than healthy controls $(100.6 \mathrm{~cm}$ vs $85.9 \mathrm{~cm} ; 26 \mathrm{~kg} / \mathrm{m}^{2}$ vs $24.3 \mathrm{~kg} / \mathrm{m}^{2}$ respectively). LVC patients had a higher WC ( $89 \mathrm{vs} 85.9 \mathrm{~cm})$, however the BMI of LVC patients was lower than healthy controls ( 21.9 vs $24.3 \mathrm{~kg} / \mathrm{m}^{2}$ ).

\subsubsection{Lipid profile}

On comparing high-dose statin treated HVC patients with the healthy controls, there were significant differences in LDL (105.1 vs $91.4 \mathrm{mg} / \mathrm{dl}$ ), LDL:HDL (3 vs 2.6) and TC:HDL (5 vs 4.3), all of which were higher, and HDL $(35.8 \mathrm{mg} / \mathrm{dl}$ vs $39.5 \mathrm{mg} / \mathrm{dl})$ which was lower in the HVC patients group. However, on comparing treated LVC patients with the healthy, except for lower HDL in LVC group ( 35.5 vs $39.5 \mathrm{mg} / \mathrm{dl}$ ), interestingly none of the lipid profile parameters showed any difference as compared to health controls (Table 3).

\subsection{Multivariate analysis}

Age, sex, smoking, diabetes and hypertension were identified as significant socio-demographic confounders from univariate analysis (Table 1). When adjusted for all of the significant socio-demographic confounders, we found that VFR, WC, HDL and the ratios of LDL:HDL and TC:HDL are independent risk factors in treated CAD patients (Table 4).
We further analyzed the association of these conventional risk factors with VFR. On adjusted multivariate analysis of healthy controls with treated LVC patients, we found that again WC, HDL and the ratios of LDL:HDL and TC:HDL are significant risk factors among treated LVC patients. On adjusted multivariate analysis of healthy controls with treated HVC patients, in addition to WC, HDL, LDL:HDL and TC:HDL, we also identified BMI and LDL as significant risk factors among treated HVC patients and are associated with VFR (Table 5).

\section{Discussion}

In the present study, a large majority were males. Male sex hormones are implicated with visceral obesity. ${ }^{7}$ Smoking is an important risk factor for $\mathrm{CAD}^{8}$ and there were a large number of current smokers among both HVC (39.2\%) and LVC patient group (42.1\%) which was disappointing to note. Diabetes, which is estimated to increase 3 fold risk of cardiovascular diseases, ${ }^{9}$ and is identified to have a relationship with visceral obesity. ${ }^{10}$ There were expectedly more diabetics among HVC patients $(56.8 \%)$ in comparison to LVC $(47.4 \%)$. The proportion of hypertensives was also higher among the HVC patients (54.1\%) in comparison to LVC patients (36.8\%). Massaro et al had also demonstrated higher visceral fat among hypertensives compared to their normal counterparts. ${ }^{11}$ Alcohol drinking beyond moderation is implicated with raised CAD risk. $^{12}$ The HVC and LVC patients had $17.6 \%$ and $14.5 \%$ alcoholics respectively.

Larger WC seen in HVC patients suggests that the fat deposition around waist region is associated with increased visceral fat accumulation as also previously reported by Ian et al. ${ }^{13}$ However, WC might sometimes be misleading when assessing risk for $\mathrm{CAD}$, as it includes both subcutaneous and visceral fat. Therefore, it is prudent to measure VFR along with WC.

Interestingly BMI of LVC patients was even lower than the controls. This pattern highlights that the effect of conventional treatment with aggressive lipid lowering and lifestyle changes on anthropometric indices might be more beneficial among CAD patients with low visceral fat. CAD patients with high visceral fat seem relatively resistant to such measures.

Table 5 - Multivariate analysis comparing healthy controls versus LVC and HVC patients adjusted for all confounding socio-demographic characteristics.

\begin{tabular}{|c|c|c|c|c|}
\hline \multirow[b]{2}{*}{ Parameters } & \multicolumn{2}{|c|}{ Healthy controls vs LVC patients } & \multicolumn{2}{|c|}{ Healthy controls vs HVC patients } \\
\hline & Odds ratio (confidence interval) & $\overline{p \text {-value }}$ & Odds ratio (confidence interval) & $p$-value \\
\hline WC (cm) & $1.07(1.01-1.14)$ & 0.016 & $1.36(1.18-1.56)$ & $<0.001$ \\
\hline BMI $\left(\mathrm{kg} / \mathrm{m}^{2}\right)$ & $0.97(0.83-1.12)$ & 0.663 & $1.37(1.13-1.61)$ & 0.002 \\
\hline $\mathrm{TG}(\mathrm{mg} / \mathrm{dl})$ & $1.00(0.99-1.01)$ & 0.485 & $1.00(1.00-1.01)$ & 0.391 \\
\hline TC (mg/dl) & $1.00(0.98-1.01)$ & 0.645 & $1.01(1.00-1.02)$ & 0.228 \\
\hline HDL (mg/dl) & $0.93(0.88-0.98)$ & 0.004 & $0.90(0.85-0.96)$ & 0.002 \\
\hline LDL (mg/dl) & $1.00(0.99-1.01)$ & 0.611 & $1.01(1.00-1.03)$ & 0.047 \\
\hline TC/HDL & $1.42(1.06-1.91)$ & 0.019 & $1.74(1.26-2.41)$ & 0.001 \\
\hline LDL/HDL & $1.49(1.05-2.12)$ & 0.026 & $1.96(1.31-2.92)$ & 0.001 \\
\hline
\end{tabular}

LVC = Low visceral fat $(\leq 12)$ CAD patients; VFR = Visceral Fat Rating; WC = Waist Circumference, BMI = Body Mass Index; TG = Triglyceride, $\mathrm{TC}=$ Total Cholesterol; HDL $=$ High Density Lipoprotein $; \mathrm{LDL}=$ Low Density Lipoprotein. 
This study shows that CAD patients with high visceral obesity have more deranged lipid profile despite aggressive treatment in comparison to their counterparts with low visceral obesity, thereby highlighting the ill effect of high visceral fat on lipid metabolism. Nguyen et al also showed that raised visceral fat is associated with dyslipidemia. ${ }^{14}$

On comparing the lipid profile, it was seen that treated HVC patients had deranged LDL, HDL, LDL:HDL and TC:HDL ratio in comparison to healthy controls while treated LVC patients were similar with healthy controls with respect to lipid profile. It was also highlighted that treated HVC patients had higher TG, TC and LDL as compared to treated LVC patients. This again emphasizes towards the inadequacy of lipid lowering treatment, among CAD patients with high visceral fat. Hence, lowering visceral fat may prove to be a crucial part of treatment plan in HVC patients.

We found that age, male sex, diabetes, smoking, hypertension, VFR, WC and HDL to be independent risk factors among treated CAD group. When all the anthropometric and lipid profile parameters were adjusted for sociodemographic parameters, only VFR, WC, HDL and HDL dependent ratios (LDL:HDL and TC:HDL) were found to be significant risk factors among CAD patients. On subgroup analysis, WC, HDL and HDL dependent ratios (LDL:HDL and TC:HDL) were also found to be significant risk factors among LVC patients group. Interestingly, in addition to WC, HDL, LDL:HDL and TC:HDL, we found risk factors of BMI and LDL were also significant among HVC patient group. This shows that treated HVC patients are associated with more risk factors for CAD in comparison to LVC patients and also implies that BMI and LDL are associated as risk factors along with VFR, further emphasizing towards the importance of VFR measurement in CAD patients. Although interventions to control visceral obesity like weight reduction and insulin resistance are under continuous research, ${ }^{15}$ none is proven significantly effective yet. There is need of further studies in this regards. Interestingly, in all study groups, HDL levels were below the normal range. ${ }^{3}$ HDL popularly known as 'good cholesterol' is found to be typically subnormal in Indian population. Therefore, aspects of visceral obesity and HDL levels must be kept in mind while planning treatment of CAD patients.

\subsection{Limitations}

Variations in environmental and clinical conditions may also affect the BIA readings. We attempted to minimize these variations by analyzing all the subjects only between 9 and 11 am and ensuring the vigorous exclusion criteria along with no food intake in the last $2 \mathrm{~h}$, no alcohol intake in last $24 \mathrm{~h}$, no intense exercise in last $2 \mathrm{~h}$, no signs of dehydration, no calluses on heals or soles and avoidance of nylon clothing; if necessary drop of isopropyl alcohol on footpads was used. The footpads were be cleaned with isopropyl alcohol after every use. However, environmental temperature and abnormal fluid overload in diseased cardiac patients may still alter the readings. There are chances of error in BIA calculations of VFR as the regression equations used were not standardized based on the population. Large scale surveys are needed in this regards.

\section{Conclusion}

CAD patients with high visceral fat have impaired anthropometric indices and lipid profile in contrast to CAD patients with visceral fat within normal lipids. Therefore, high visceral fat, in addition to being an independent risk factor of CAD, is also associated with deranged conventional risk factors. CAD patients with high visceral fat are also associated with additional conventional risk factors of BMI and LDL in comparison to $C A D$ patients with low visceral fat. Moreover, we hypothesize that CAD patients with high visceral fat fail to show improvement in anthropometric indices and HDL levels on conventional treatment along with aggressive lipid lowering therapy. On the other hand, with the same treatment, CAD patients with low visceral fat have anthropometric and lipid profile similar to healthy controls, thereby indicating adequacy of standard treatment in low visceral fat patients. Hence, high visceral fat accumulation can be considered as an indicator of raised future risk of $\mathrm{CAD}$ and poor response to treatment. It is thus very important to monitor visceral fat along with the conventional indices. Treatment of visceral fat may prove to be a key approach for a large population of CAD patients and warrants for further research.

\section{Conflicts of interest}

All authors have none to declare.

\section{Acknowledgment}

This work was supported under the intramural seed grant 2012 provided by Research Cell, King George Medical University and we would like to make a special mention about our Faculty In Charge, Dr S. Awasthi who also guided us in this project. We are thankful to Director, Institute for Data Computing and Training (I.D.C.T.), Lucknow for providing valuable assistance in data analysis.

\section{R E F E R E N C E S}

1. Tsujinaka S, Konishi F, Kawamura Y, et al. Visceral obesity predicts surgical outcomes after laparoscopic colectomy for sigmoid colon cancer. Dis Colon Rectum. 2008;51:1757-1767.

2. Houtkooper LB, Lohman TG, Going SB, Howell WH. Why bioelectrical impedance analysis should be used for estimating adiposity. Am J Clin Nutr. 1996;64:436S-448S.

3. Grundy Sm BD, Clark LT, on behalf of the National Cholesterol Education Program (NCEP) Expert Panel on Detection, Evaluation, and Treatment of High Blood Cholesterol in Adults (Adult Treatment Panel III). Third report of the National Cholesterol Education Program (NCEP) expert panel on detection, evaluation, and treatment of high blood cholesterol in adults (Adult Treatment Panel III). Bethesda (MD): National Institutes of Health 2002. Circulation. 2002;106:3143-3421.

4. Wilson PWF, D'Agostino RB, Levy D, Belanger AM, Silbershatz H, Kannel WB. Prediction of coronary heart 
disease using risk factor Categories. Circulation. 1998;97:1837-1847.

5. Grundy S, Bilheimer D, Chait A, et al. Summary of the second report of the national cholesterol education program expert panel on detection, evaluation, and treatment of high blood cholesterol in adults. J Am Med Assoc. 1993;269:3015-3023.

6. Chumlea WC, Baumgartner RN, Roche AF. Specific resistivity used to estimate fat-free mass from segmental body measures of bioelectric impedance. Am J Clin Nutr. 1988;48:7-15.

7. Carr MC, Hokanson JE, Zambon A, et al. The contribution of intraabdominal fat to gender differences in hepatic lipase activity and low/high density lipoprotein heterogeneity. Am J Physiol Endocrinol Metab. 2001;86:2831-2837.

8. Chiolero A, Faeh D, Paccaud F, Cornuz J. Consequences of smoking for body weight, body fat distribution, and insulin resistance. Am J Clin Nutr. 2008;87:801-809.

9. Yusuf S, Hawken S, Ônpuu S, et al. Obesity and the risk of myocardial infarction in 27000 participants from 52 countries: a case-control study. Lancet. 2005;366:1640-1649.
10. Després J-P. The insulin resistance-dyslipidemic syndrome of visceral obesity: effect on patients' risk. Obes Res. 1998;6:8S-17S.

11. Pou KM, Massaro JM, Hoffmann U, et al. Patterns of abdominal fat distribution. Diabetes Care. 2009;32:481-485.

12. Roerecke $\mathrm{M}, \mathrm{Rehm} J$. Irregular heavy drinking occasions and risk of ischemic heart disease: a systematic review and metaanalysis. Am J Epidemiol. 2010;171:633-644.

13. Janssen I, Heymsfield SB, Allison DB, Kotler DP, Ross R. Body mass index and waist circumference independently contribute to the prediction of nonabdominal, abdominal subcutaneous, and visceral fat. Am J Clin Nutr. 2002;75:683-688.

14. Nguyen-Duy T-B, Nichaman MZ, Church TS, Blair SN, Ross R. Visceral fat and liver fat are independent predictors of metabolic risk factors in men. Am J Physiol Endocrinol Metab. 2003;284:E1065-E1071.

15. Brochu M, Tchernof A, Turner AN, Ades PA, Poehlman ET. Is there a threshold of visceral fat loss that improves the metabolic profile in obese postmenopausal women? Metabolism. 2003;52:599-604. 